Pathologe 2019 - 40 (Suppl 3):S417-S418 https://doi.org/10.1007/s00292-019-00652-3 Online publiziert: 28. Oktober 2019

(c) Springer Medizin Verlag GmbH, ein Teil von Springer Nature 2019
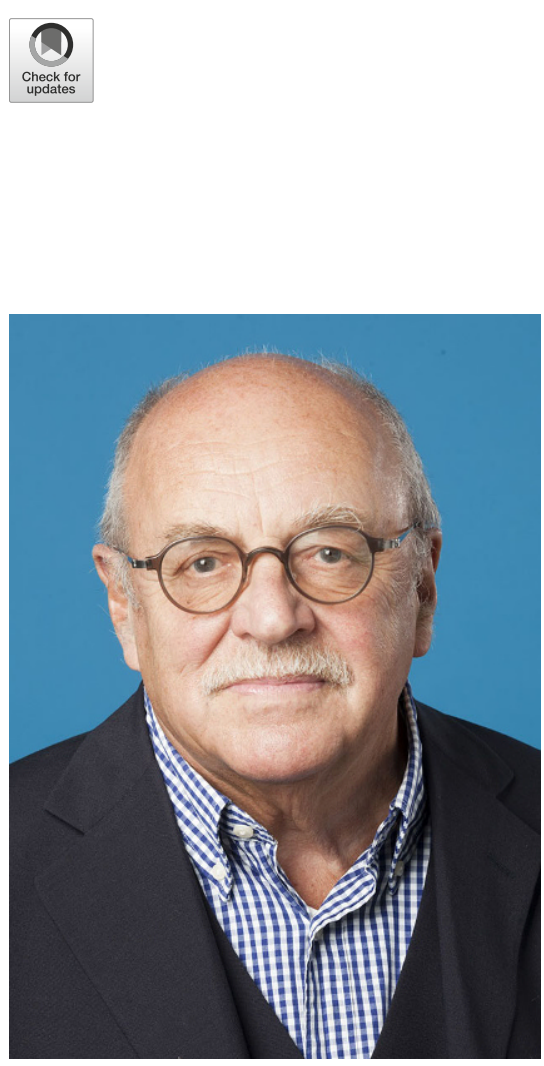

Peter Meister

Prof. Dr. med. Peter Meister - einer der meistgekannten und allseits geschätzten Kollegen unseres Fachgebiets - ist am 23. März 2019 in München verstorben.

Peter Meister war Oberfranke. Er wurde am 12. Mai 1931 in Bayreuth geboren und wuchs als Sohn eines praktischen Arztes in der Kleinstadt Kulmbach auf, wo er 1950 das Abitur ablegte. Wegweisend für seine spätere Weltoffenheit und sein nichtprovinzielles Denken war wahrscheinlich eine glückliche Fügung. Bei seinen Bewerbungen um einen Medizinstudienplatz erhielt er Anfang der 1950er-Jahre von renommierten deutschen Universitäten keine Antwort, sondern nur aus der Universität Zürich einen positiven Bescheid. Damit konnte er dem zerstörten und versehrten Nachkriegsdeutschland entfliehen und im unbeschädigten und weltgewandten Zürich seine Ausbildung in Medizin beginnen.

\title{
T. Kirchner
}

Pathologisches Institut, Ludwig-Maximilians-Universität München, München, Deutschland

\section{Peter Meister}

\subsubsection{1-23.03.2019}

In Zürich, wo er bis auf ein Semester in Genf sein gesamtes Studium absolvierte, wurden zwei Begegnungen bestimmend für seinen weiteren Weg: Zum einen erlebte er in der Vorlesung den Züricher Lehrstuhlinhaber für Pathologie, Erwin Uehlinger, als einen herausragenden Fachvertreter und berühmten Knochentumorforscher, bei dem er promovierte und durch den sein langjähriges Interesse an den Sarkomen geweckt wurde. Zum zweiten gab es in Zürich amerikanische Studienkollegen, mit denen er sich anfreundete und Beziehungen zum Gesundheitssystem der USA knüpfte. Beides bewirkte, dass sich Peter Meister nach der Medizinalassistentenzeit in Deutschland entschloss, 1959 in die USA aufzubrechen und am Cook County Hospital in Chicago - einem der damals drei größten amerikanischen Krankenhäuser - seine gesamte Weiterbildung in Pathologie zu absolvieren.

Erst 1966 kehrte Peter Meister als fertiger „senior pathologist“ nach Deutschland zurück. Hier fand er Aufnahme am Pathologischen Institut der Universität München bei dem damaligen Lehrstuhlinhaber Walter Büngeler, der als Kosmopolit selbst mehrere Jahre in Sao Paulo (Brasilien) tätig gewesen war. Walter Büngeler integrierte Peter Meister in das deutsche System und förderte dessen $\mathrm{Ha}$ bilitation für Allgemeine Pathologie und Pathologische Anatomie, die 1969 erfolgte. Max Eder - Büngelers Nachfolger auf dem Münchener Lehrstuhl für Pathologie - setzte diese Unterstützung fort, sodass Peter Meister 1975 zum außerplanmäßigen Professor und darauf zum Extraordinarius avancierte. Im September 1981 wurde Peter Meister dann die Leitung des Instituts für Pathologie am Städtischen Krankenhaus München Har- laching übertragen, wo er 15 Jahre bis 1996 als Chefarzt tätig war.

Das Medizinstudium in Zürich und die Facharztweiterbildung in Chicago haben das Denken und die Arbeit von Peter Meister in der Pathologie geprägt und bis zuletzt nachhaltig bestimmt. Sein Ideal war eine stark klinisch ausgerichtete Pathologie und deren teamorientierte Verwirklichung in enger Zusammenarbeit mit den klinischen Kollegen. In den 1960er-Jahren brachte Peter Meister daher frischen Wind und wichtige neue Impulse in die deutsche Pathologie. Mit ihm starteten am Münchener Institut Biopsie- und Tumorkonferenzen, wie sie heute Standard in allen Einrichtungen für Pathologie sind.

Bei seinem amerikanischen Pathologielehrer Paul Szanto, der eng mit dem berühmten Pathologen Hans Popper in Chicago zusammenarbeitete, fand Peter Meister einen wissenschaftlichen und klinischen Zugang zur Leberpathologie. In München setzte er die in den USA begonnenen Arbeiten zur Leberzirrhose und $\mathrm{zu}$ alkoholischen Lebererkrankungen fort. Zugleich startete er einen engen klinisch-pathologischen Austausch mit dem Münchener Hepatologen Josef Eisenburg in Form von wissenschaftlichen Kooperationen und Biopsiekonferenzen zu den damals stark aufkommenden Leberpunktionen. Hinzu kamen wissenschaftliche Untersuchungen zur Glomerulonephritis und zur orthopädischen Pathologie sowie Biopsiekonferenzen zum Magen-Darm-Trakt, zur Dermatopathologie oder zu den zunehmend durchgeführten Herzbiopsien.

Das Hauptthema der wissenschaftlichen und klinisch-pathologischen Arbeiten von Peter Meister wurden jedoch ab den späten 1960er- und den frühen 
1970er-Jahren die Weichgewebstumoren. Er nahm die neuen Klassifikationsvorschläge von Arthur Purdy Stout und Franz Enzinger aus den USA auf und machte deren Tumortypisierung auch für die diagnostische Praxis in Deutschland populär und nutzbar. Als prägender und wegweisender Pathologe hat Peter Meister ab 1976 zu den Weichgewebstumoren sechs große Symposien und Tutorials der deutschen Sektion der Internationalen Akademie für Pathologie (IAP) durchgeführt. Zudem war er maßgeblich beteiligt an 21 weiteren IAPSymposien und Tutorials insbesondere zur Pathologie der Haut, aber auch zu verschiedenen anderen Themen. Zuletzt hat Peter Meister 2015 im Alter von 83 Jahren gemeinsam mit Erhard Bierhoff erneut ein IAP-Symposion zu den „Neoplasien der Haut“ gestaltet. Und in 2018 war er noch einmal aktiv am traditionellen Baltisch-Deutschen IAPSymposium in Estland beteiligt.

In der Deutschen Gesellschaft für $\mathrm{Pa}$ thologie war Peter Meister 8 Jahre lang von 2008 bis 2016 - der Vorsitzende der Arbeitsgemeinschaft für Dermatopathologie. Er war ein wichtiger Mitinitiator der deutsch-spanischen Pathologentreffen und Dauermitglied der von seinem Doktorvater Erwin Uehlinger gegründeten Arbeitsgemeinschaft für Knochentumoren. Seine stärkste Verbundenheit galt jedoch der Internationalen Akademie für Pathologie (IAP), da diese für Peter Meister die wichtigste Plattform zum internationalen Austausch der Pathologen und die enge Verbindung zur US-amerikanischen und kanadischen Pathologie darstellte. Von 1996 bis 1998 hat Peter Meister mit großer Umsicht und Weitsicht als Präsident die deutsche Sektion der IAP geführt. Im Jahr 2000 wurde ihm die Ehrenmitgliedschaft der IAP für seine hohen Verdienste um die Akademie verliehen. Seine langjährigen Kontakte in der IAP haben mit dazu beigetragen, dass der 26. Weltkongress der IAP 2016 in Deutschland stattfinden konnte.

Trotz des nationalen und internationalen Engagements und bei aller Weltoffenheit bewahrte Peter Meister stets die Bodenhaftung und eine tiefe Ortsverbundenheit zu München, das seit 1966 - also 53 Jahre - sein berufliches und privates
Zentrum war. Mit viel Engagement und hoher Kompetenz hat er die Pathologie in Harlaching geleitet und über München hinaus bekannt gemacht. Bis zuletzt hat er stets an den Treffen der Bayerischen und Münchener Pathologen teilgenommen und unseren Gemeinschaftssinn wesentlich gefördert. Dabei befand er sich auch noch im Alter von 87 Jahren mit einem offenen Ohr stets am Puls der Zeit. Ohne Anbiederung hat er Kollegen immer wieder durch seine Erfahrung und klugen Empfehlungen geholfen. Für viele wurde er dadurch zu einem ehrlichen Ratgeber und kollegialen Freund.

Sein plötzlicher und unerwarteter Tod hat uns alle überrascht. Da er bei vielen Pathologentreffen und Tagungen auch noch im hohen Alter und bis zuletzt immer präsent war, ist es schwer zu fassen, dass er auf einmal nicht mehr da sein wird. Eigentlich stand er, der sichtbar nicht alterte, außer der Zeit. Mit seiner unverwechselbaren Art und unnachahmlichen Persönlichkeit wird er in unserer Erinnerung unvergänglich erhalten bleiben.

\section{Thomas Kirchner}

München

\section{Korrespondenzadresse}

Prof. Dr. med. T. Kirchner

Pathologisches Institut, Ludwig-Maximilians-

Universität München

Thalkirchner Str. 36, 80337 München,

Deutschland

Thomas.Kirchner@med.uni-muenchen.de

The supplement containing this article is not sponsored by industry. 